\title{
Characterizations of graded rings over which every graded semi-primary ideal is graded 1-absorbing primary
}

\author{
Alaa Melhem¹, Malik Bataineh², Rashid Abu-Dawwas ${ }^{3 *}$
}

\begin{abstract}
Let $G$ be a group with identity $e$ and $R$ be a commutative $G$-graded ring with nonzero unity 1 . Graded semiprimary and graded 1-absorbing primary ideals have been investigated and examined by several authors as generalizations of graded primary ideals. However, these three concepts are different. In this article, we characterize graded rings over which every graded semi-primary ideal is graded 1-absorbing primary and graded rings over which every graded 1-absorbing primary ideal is graded primary.

Keywords

Graded 1-absorbing primary ideals - Graded primary ideals - Graded semi-primary ideals

${ }^{1}$ Department of Mathematics, University of Jordan, Amman, Jordan

${ }^{2}$ Department of Mathematics and Statistics, Jordan University of Science and Technology, Irbid, Jordan

${ }^{3}$ Department of Mathematics, Yarmouk University, Irbid, Jordan

*Corresponding author: rrashid@yu.edu.jo
\end{abstract}

\section{Contents}

\section{Introduction}

1 Graded rings over which every graded semi-primary ideal is graded 1-absorbing primary

References

\section{Introduction}

Throughout this article, all rings are commutative with nonzero unity 1 . Let $G$ be a group with identity $e$. Then a ring $R$ is said to be $G$-graded if $R=\bigoplus_{g \in G} R_{g}$ with $R_{g} R_{h} \subseteq R_{g h}$ for all $g, h \in G$, where $R_{g}$ is an additive subgroup of $R$ for all $g \in G$. The elements of $R_{g}$ are called homogeneous of degree $g$. If $x \in R$, then $x$ can be written uniquely as $\sum_{g \in G} x_{g}$, where $x_{g}$ is the component of $x$ in $R_{g}$. Also, we set $h(R)=\bigcup_{g \in G} R_{g}$. Moreover, it has been proved in [9] that $R_{e}$ is a subring of $R$ and $1 \in R_{e}$. Let $I$ be an ideal of a graded ring $R$. Then $I$ is said to be a graded ideal if $I=\bigoplus_{g \in G}\left(I \cap R_{g}\right)$, i.e., for $x \in I, x=\sum_{g \in G} x_{g}$, where $x_{g} \in I$ for all $g \in G$. An ideal of a graded ring need not be graded (see [9]).

Let $P$ be a proper graded ideal of $R$. Then the graded radical of $P$ is $\operatorname{Grad}(P)$ and is defined as follows: $x \in \operatorname{Grad}(P)$ if and only if for every $g \in G$, there exists $n_{g} \in \mathbb{N}$ such that $x_{g}^{n_{g}} \in P$. Note that $\operatorname{Grad}(P)$ is always a graded ideal of $R$ (see [12]).
Proposition 0.1. ([8]) Let $R$ be a G-graded ring.

1. If $I$ and $J$ are graded ideals of $R$, then $I+J, I J$ and $I \cap J$ are graded ideals of $R$.

2. If $a \in h(R)$, then Ra is a graded ideal of $R$.

Let $R$ be a $G$-graded ring and $I$ be a graded ideal of $R$. Then $R / I$ is a $G$-graded ring by $(R / I)_{g}=\left(R_{g}+I\right) / I$ for all $g \in G$. Moreover, if $P$ is an ideal of $R$ containing $I$, then $P$ is a graded ideal of $R$ if and only if $P / I$ is a graded ideal of $R / I$, see ([13], Lemma 3.2).

Let $R$ and $S$ be two $G$-graded rings. Then $T=R \times S$ is a $G$-graded ring by $T_{g}=R_{g} \times S_{g}$ for all $g \in G$. Furthermore, $P \times K$ is a graded ideal of $R \times S$ if and only if $P$ is a graded ideal of $R$ and $K$ is a graded ideal of $S$ ([6], Lemma 4).

If $R$ is a $G$-graded ring and $S \subseteq h(R)$ is a multiplicative set, then $S^{-1} R$ is a $G$-graded ring with $\left(S^{-1} R\right)_{g}=$

$\left\{\frac{a}{s}, a \in R_{h}, s \in S \cap R_{h g^{-1}}\right\}$ for all $g \in G$. Moreover, if $I$ is a graded ideal of $R$, then $S^{-1} I$ is a graded ideal of $S^{-1} R$ [9].

Graded primary ideals have been introduced by Refai and Al-Zoubi in [11]. A proper graded ideal $Q$ of $R$ is said to be graded primary if whenever $x, y \in h(R)$ with $x y \in Q$, then $x \in Q$ or $y \in \operatorname{Grad}(Q)$. In this case $P=\operatorname{Grad}(Q)$ is a graded prime ideal of $R$, and $Q$ is said to be graded $P$-primary. In [4], Al-Zoubi and Sharafat introduced a generalization of graded primary ideals called graded 2-absorbing primary ideals. A proper graded ideal $I$ of $R$ is called a graded 2-absorbing primary ideal of $R$ if whenever $x, y, z \in h(R)$ and $x y z \in I$, then $x y \in I$ or $x z \in \operatorname{Grad}(I)$ or $y z \in \operatorname{Grad}(I)$. The concept of graded 
2-absorbing primary ideals is generalized in many ways, for example, see [3, 15]. Recently in [7], we consider a new class of graded ideals called the class of graded 1-absorbing primary ideals. A proper graded ideal $P$ of $R$ is said to be a graded 1 -absorbing primary ideal of $R$ if whenever nonunit elements $x, y, z \in h(R)$ such that $x y z \in P$, then $x y \in P$ or $z \in \operatorname{Grad}(P)$. Clearly, every graded primary ideal is graded 1 -absorbing primary ideal. The next example shows that the converse is not true in general.

Example 0.2. Consider $R=K[X, Y]$, where $K$ is a field, and $G=\mathbb{Z}$. Then $R$ is G-graded by $R_{n}=\bigoplus_{i+j=n, i, j \geq 0} K X^{i} Y^{j}$ for all $n \in \mathbb{Z}$. Note that deg $(X)=\operatorname{deg}(Y)=1$. Consider the graded ideal $P=\left\langle X^{2}, X Y\right\rangle$ of $R$. Then $\operatorname{Grad}(P)=\langle X\rangle$. Since for $X . Y . X \in P$, either $X . Y \in P$ or $X \in \operatorname{Grad}(P), P$ is a graded 1-absorbing primary ideal of $R$. On the other hand, $P$ is not graded primary ideal of $R$ by ([14], Example 2.11).

Also, it is clear that every graded 1-absorbing primary ideal is graded 2-absorbing primary ideal. The next example shows that the converse is not true in general.

Example 0.3. Let $R=\mathbb{Z}[i]$ and $G=\mathbb{Z}_{2}$. Then $R$ is $G$-graded by $R_{0}=\mathbb{Z}$ and $R_{1}=i \mathbb{Z}$. Consider $P=12 R$. Then as $12 \in$ $h(R), P$ is a graded ideal of $R$. By ([4], Example 2.2 (ii)), $P$ is a graded 2-absorbing primary ideal of $R$. On the other hand, $2,3 \in h(R)$ such that $2.2 .3 \in P$, but neither $2.2 \in P$ nor $3 \in \operatorname{Grad}(P)$. So, $P$ is not graded 1-absorbing primary ideal of $R$.

So, we can state the following:

graded primary $\underbrace{\Rightarrow}_{1}$ graded 1-absorbing primary $\underbrace{\Rightarrow}_{2}$ graded

And the arrows (1) and (2) are irreversible by Example 0.2 and Example 0.3.

In [6], a proper graded ideal $P$ of $R$ is said to be a graded semi-primary ideal of $R$ if whenever $x, y \in h(R)$ such that $x y \in P$, then either $x \in G \operatorname{rad}(P)$ or $y \in G \operatorname{Grad}(P)$. In this article, we prove that $P$ is a graded semi-primary ideal of $R$ if and only if $\operatorname{Grad}(P)$ is a graded prime ideal of $R$ (Proposition 1.1). Then we show that if $P$ is a graded 1-absorbing primary ideal of $R$, then $P$ is a graded semi-primary ideal of $R$ (Proposition 1.2). After that, we prove that if $R$ is a principal ideal domain, then the three concepts; graded primary, graded semi-primary and graded 1-absorbing primary ideals will be equivalent (Proposition 1.4).

Motivated by Proposition 1.4, our goal in this article is following [2] to characterize graded rings over which every graded semi-primary ideal is graded 1-absorbing primary and graded rings over which every graded 1 -absorbing primary ideal is graded primary.

\section{Graded rings over which every graded semi-primary ideal is graded 1 -absorbing primary}

In this section, we characterize graded ring $R$ over which every graded semi-primary ideal is graded 1 -absorbing primary. The next proposition has been proved in ([6], Lemma 1$)$ for $\mathbb{Z}$ graded rings. In fact, it is true for any $G$-graded ring.

Proposition 1.1. Let $R$ be a graded ring and $P$ be a graded ideal of $R$. Then $P$ is a graded semi-primary ideal of $R$ if and only if $G r a d(P)$ is a graded prime ideal of $R$.

Proof. Suppose that $P$ is a graded semi-primary ideal of $R$. Let $x, y \in h(R)$ such that $x y \in G r a d(P)$. Then $(x y)^{n}=x^{n} y^{n} \in P$ for some positive integer $n$. Since $P$ is graded semi-primary, we have $x^{n} \in \operatorname{Grad}(P)$ or $y^{n} \in \operatorname{Grad}(P)$, and then $x^{n k} \in P$ or $y^{n k} \in P$ for some positive integer $k$, which implies that $x \in$ $\operatorname{Grad}(P)$ or $y \in \operatorname{Grad}(P)$. Hence, $\operatorname{Grad}(P)$ is a graded prime ideal of $R$. Conversely, let $x, y \in h(R)$ such that $x y \in P$. Then as $P \subseteq \operatorname{Grad}(P), x y \in \operatorname{Grad}(P)$. Since $\operatorname{Grad}(P)$ is graded prime, we have $x \in \operatorname{Grad}(P)$ or $y \in \operatorname{Grad}(P)$. Hence, $P$ is a graded semi-primary ideal of $R$.

Proposition 1.2. Let $R$ be a graded ring and $P$ be a graded ideal of $R$. If $P$ is a graded 1-absorbing primary ideal of $R$, then $P$ is a graded semi-primary ideal of $R$.

Proof. Let $a, b \in h(R)$ such that $a b \in G r a d(P)$. We may assume that $a, b$ are nonunit elements of $R$. Let $k \geq 2$ be an even positive integer such that $(a b)^{k} \in P$. Then $k=2 s$ for some positive integer $s \geq 1$. Since $(a b)^{k}=a^{k} b^{k}=a^{s} a^{s} b^{k} \in P$ and $P$ is a graded 1-absorbing primary ideal of $R$, we conclude that $a^{s} a^{s}=a^{k} \in P$ or $b^{k} \in P$. Hence, $a \in \operatorname{Grad}(P)$ or $b \in \operatorname{Grad}(P)$. Thus $\operatorname{Grad}(P)$ is a graded prime ideal of $R$, and then $P$ is a graded semi-primary ideal of $R$ by Proposition 1.1 .

Corollary 1.3. Let $R$ be a graded ring and $P$ be a graded ideal of $R$. If $P$ is a graded 1-absorbing primary ideal of $R$, then $\operatorname{Grad}(P)$ is a graded prime ideal of $R$.

Proof. Apply Proposition 1.1 and Proposition 1.2.

Totally, we can state the following:

graded primary $\underbrace{\Rightarrow}_{3}$ graded 1-absorbing primary $\underbrace{\Rightarrow}_{\text {semi-primary. }}$ graded

By Example 0.2, arrow (3) is irreversible. Now, let $R$ be a graded ring and $P$ be a graded 1 -absorbing primary ideal of $R$ which is not graded primary. Set $T=R \times R$ and $Q=$ $P \times R$. Then $Q$ is a graded ideal of $T$ by ([6], Lemma 4) with $\operatorname{Grad}(Q)=\operatorname{Grad}(P \times R)=\operatorname{Grad}(P) \times R$ is a graded prime ideal of $T$ since $\operatorname{Grad}(P)$ is a graded prime ideal of $R$ by Corollary 1.3. Thus, $Q$ is a graded semi-primary ideal of $T$ by Proposition 1.1. On the other hand, it is well known that graded primary ideals of $T$ are $I \times R$ and $R \times I$ with $I$ is a 
graded primary ideal of $R$. Hence, $Q$ is not a graded primary ideal of $T$. In fact, we could not give an example showing that arrow (4) is irreversible. However, if $R$ is a principal ideal domain, then the three concepts will be equivalent as one can see in the next proposition that should be compared with ([6], Proposition 2).

Proposition 1.4. Let $R$ be a principal ideal domain. If $R$ is graded and $P$ is a graded ideal of $R$, then the following statements are equivalent:

\section{1. $P$ is a graded primary ideal of $R$.}

2. $P$ is a graded 1-absorbing primary ideal of $R$.

3. $P$ is a graded semi-primary ideal of $R$.

Proof. $(1) \Rightarrow(2)$ : Clear.

$(2) \Rightarrow(3)$ : Proposition 1.2.

$(3) \Rightarrow(1)$ : Suppose that $P$ is a graded semi-primary ideal of $R$. Then by Proposition 1.1, $\operatorname{Grad}(P)$ is a graded prime ideal, and then as $R$ is a principal ideal domain, $\operatorname{Grad}(P)$ is a graded maximal ideal of $R$, which implies that $P$ is a graded primary ideal of $R$ by ([11], Proposition 1.11).

In [13], a graded ring $R$ is said to be a graded domain if it has no homogeneous zero divisors. Clearly, every domain is a graded domain, but the converse is not necessarily true, see ([13], Example 3.6).

Proposition 1.5. Let $R$ be a graded domain. If every nonzero graded prime ideal of $R$ is graded maximal, then every graded semi-primary ideal of $R$ is graded primary.

Proof. Let $P$ be a graded semi-primary ideal of $R$. If $P=\{0\}$, then $P$ is a graded prime ideal of $R$, and then $P$ is a graded primary ideal of $R$. If $P \neq\{0\}$, then $\operatorname{Grad}(P)$ is a nonzero graded prime ideal of $R$ by Proposition 1.1, and then $\operatorname{Grad}(P)$ is a graded maximal ideal of $R$ by assumption, and hence $P$ is a graded primary ideal of $R$ by ([11], Proposition 1.11).

Corollary 1.6. Let $R$ be a graded domain. If every nonzero graded prime ideal of $R$ is graded maximal, then every graded semi-primary ideal of $R$ is graded 1-absorbing primary.

Clearly, if every graded semi-primary ideal is graded primary, then every graded semi-primary ideal is graded 1absorbing primary. However, we give an example that introduces a case where every graded semi-primary ideal is graded 1-absorbing primary, but there is a graded semi-primary ideal which is not graded primary:

Example 1.7. Consider $T=K[X, Y]$, where $K$ is a field, and $G=\mathbb{Z}$. Then $T$ is $G$-graded by $T_{n}=\bigoplus_{i+j=n, i, j \geq 0} K X^{i} Y^{j}$ for all $n \in \mathbb{Z}$. Consider the graded ideal $J=\left\langle X^{2}, X Y\right\rangle$ of $T$. Then $R=T / J$ is a graded ring and $P=\langle X, Y\rangle / J$ is a graded prime ideal of $R$. So, $S=h(R)-P$ is a multiplicative subset of $h(R)$, and then $S^{-1} R$ is a graded local ring with graded maximal ideal $M=\left\langle\frac{\bar{X}}{\overline{1}}, \frac{\bar{Y}}{\overline{1}}\right\rangle$. Note that, $P=\operatorname{Grad}\left(\left\{0_{S^{-1} R}\right\}\right)=\left\langle\frac{\bar{X}}{\overline{1}}\right\rangle$ is a graded prime ideal of $S^{-1} R$ which is not graded maximal, and $P M=\left\{0_{S^{-1} R}\right\}$. Let I be a graded semi-primary ideal of $S^{-1} R$. Then Grad $(I)$ is a graded prime ideal of $S^{-1} R$ by Proposition 1.1, and then either $\operatorname{Grad}(I)=M$ or $\operatorname{Grad}(I)=$ $\operatorname{Grad}\left(\left\{0_{S^{-1} R}\right\}\right)$. If $\operatorname{Grad}(I)=M$, then $I$ is graded primary by ([11], Proposition 1.11), and so I is graded 1-absorbing primary. Suppose that $\operatorname{Grad}(I)=\operatorname{Grad}\left(\left\{0_{S^{-1} R}\right\}\right)$. Assume that $a, b, c \in h\left(S^{-1} R\right)$ be nonunit elements such that abc $\in I$ and $c \notin \operatorname{Grad}\left(\left\{0_{S^{-1} R}\right\}\right)$. Then either $a \in \operatorname{Grad}\left(\left\{0_{S^{-1} R}\right\}\right)$ or $b \in \operatorname{Grad}\left(\left\{0_{S^{-1} R}\right\}\right)$, which implies in both cases that $a b=$ $0_{S^{-1} R} \in I$. Hence, I is a graded 1-absorbing primary ideal of $S^{-1} R$. Consequently, every graded semi-primary ideal of $S^{-1} R$ is graded 1-absorbing primary. On the other hand, $\operatorname{Grad}\left(\left\{0_{S^{-1} R}\right\}\right)$ is a graded prime ideal of $S^{-1} R$, so $\left\{0_{S^{-1} R}\right\}$ is a graded semi-primary ideal of $S^{-1} R$ by Proposition 1.1, but $\left\{0_{S^{-1} R}\right\}$ is not a graded primary ideal of $S^{-1} R$ since $\frac{\bar{X}}{\overline{1}}, \frac{\bar{Y}}{\overline{1}} \in$ $h\left(S^{-1} R\right)$ with $\frac{\bar{X}}{\overline{1}} \cdot \frac{\bar{Y}}{\overline{1}}=0_{S^{-1} R}, \frac{\bar{X}}{\overline{1}} \neq 0_{S^{-1} R}$ and $\frac{\bar{Y}}{\overline{1}} \notin \operatorname{Grad}\left(\left\{0_{S^{-1} R}\right\}\right)$.

Let $R$ and $S$ be two $G$-graded rings. In [9], a ring homomorphism $f: R \rightarrow S$ is said to be graded homomorphism if $f\left(R_{g}\right) \subseteq S_{g}$ for all $g \in G$.

Proposition 1.8. [7] Let $R$ and $S$ be G-graded rings and $f: R \rightarrow S$ be a graded homomorphism such that $f\left(1_{R}\right)=1_{S}$. Then the following hold:

1. If $K$ is a graded 1-absorbing primary ideal of $S$ and $f(x)$ is a nonunit element of $S$ for every nonunit element $x$ of $R$, then $f^{-1}(K)$ is a graded 1-absorbing primary ideal of $R$.

2. If $P$ is a graded 1-absorbing primary ideal of $R$ and $f$ is surjective with $\operatorname{Ker}(f) \subseteq P$, then $f(P)$ is a graded 1-absorbing primary ideal of $S$.

Proof. 1. By ([10], Lemma $3.11(1)), f^{-1}(K)$ is a graded ideal of $R$. Let $x, y, z \in h(R)$ be nonunit elements such that $x y z \in f^{-1}(K)$. Then $f(x), f(y), f(z) \in h(S)$ are nonunit elements such that $f(x) f(y) f(z)=f(x y z) \in K$. Since $K$ is a graded 1-absorbing primary ideal of $S$, we have that $f(x y)=f(x) f(y) \in K$ or $f(z) \in \operatorname{Grad}(K)$, which implies that $x y \in f^{-1}(K)$ or $z \in f^{-1}(\operatorname{Grad}(K))=$ $\operatorname{Grad}\left(f^{-1}(K)\right)$. Thus, $f^{-1}(K)$ is a graded 1-absorbing primary ideal of $R$.

2. By ([10], Lemma $3.11(2)), f(P)$ is a graded ideal of $S$. Let $a, b, c \in h(S)$ be nonunit elements such that $a b c \in$ $f(P)$. Then since $f$ is surjective, there exist nonunit elements $x, y, z \in h(R)$ such that $f(x)=a, f(y)=b$ and $f(z)=c$. Now, $f(x y z)=f(x) f(y) f(z)=a b c \in f(P)$. Since $\operatorname{Ker}(f) \subseteq P$, we have that $x y z \in P$. Since $P$ is a graded 1-absorbing primary ideal of $R$, we have that $x y \in P$ or $z \in \operatorname{Grad}(P)$, which implies that $a b=$ $f(x) f(y)=f(x y) \in f(P)$ or $c=f(z) \in f(\operatorname{Grad}(P))=$ $\operatorname{Grad}(f(P))$ as $f$ is surjective and $\operatorname{Ker}(f) \subseteq P$. Hence, $f(P)$ is a graded 1-absorbing primary ideal of $S$. 
Corollary 1.9. [7] Let $P$ and $K$ be proper graded ideals of a graded ring $R$ with $K \subseteq P$. If $U(R / K)=\{a+K: a \in U(R)\}$, then $P$ is a graded 1-absorbing primary ideal of $R$ if and only if $P / K$ is a graded 1-absorbing primary ideal of $R / K$.

Proof. Define $f: R \rightarrow R / K$ by $f(x)=x+K$. Then $f$ is surjective graded homomorphism and $f\left(1_{R}\right)=1_{R / K}$. Suppose that $P$ is a graded 1 -absorbing primary ideal of $R$. Since $f$ is surjective and $\operatorname{Ker}(f)=K \subseteq P$, by Proposition 1.8 (2), we have that $f(P)=P / K$ is a graded 1-absorbing primary ideal of $R / K$. Conversely, $f^{-1}(P / K)=P$ is a graded 1 -absorbing primary ideal of $R$ by Proposition $1.8(1)$.

Proposition 1.10. Let $R$ be a graded ring and $K$ be a graded ideal of $R$ such that $U(R / K)=\{a+K: a \in U(R)\}$. If every graded semi-primary ideal of $R$ is graded 1-absorbing primary, then every graded semi-primary ideal of $R / K$ is graded 1-absorbing primary.

Proof. Let $P / K$ be a graded semi-primary ideal of $R / K$. Then $\operatorname{Grad}(P / K)=\operatorname{Grad}(P) / K$ is a graded prime ideal of $R / K$ by Proposition 1.1, and then $\operatorname{Grad}(P)$ is a graded prime ideal of $R$, so $P$ is a graded semi-primary ideal of $R$ by Proposition 1.1 . Hence, $P$ is a graded 1 -absorbing primary ideal of $R$ by assumption. Therefore, $P / K$ is a graded 1 -absorbing primary ideal of $R / K$ by Corollary 1.9 .

Lemma 1.11. Let $R$ be a graded ring such that every homogeneous element is either unit or nilpotent. Then $R$ has exactly one graded prime ideal, and hence $R$ is a graded local ring with graded maximal ideal Grad $(\{0\})$.

Proof. Let $P$ be a graded prime ideal of $R$. Then $\operatorname{Grad}(\{0\}) \subseteq$ $P$. Assume that $x \in P$. Then $x_{g} \in P$ for all $g \in G$ as $P$ is a graded ideal, and then $x_{g}$ is nilpotent for all $g \in G$, which implies that $x_{g} \in \operatorname{Grad}(\{0\})$ for all $g \in G$, and so $x \in \operatorname{Grad}(\{0\})$. Therefore, $P \subseteq \operatorname{Grad}(\{0\})$, and hence $P=\operatorname{Grad}(\{0\})$. So, $\operatorname{Grad}(\{0\})$ is the only graded prime ideal of $R$. Since every graded maximal ideal is graded prime, $\operatorname{Grad}(\{0\})$ is the only graded maximal ideal of $R$. Hence, $R$ is a graded local ring with graded maximal ideal $\operatorname{Grad}(\{0\})$.

Theorem 1.12. Let $R$ be a graded ring such that every homogeneous element is either unit or nilpotent. Then every graded semi-primary ideal of $R$ is graded primary.

Proof. By Lemma 1.11, Grad $(\{0\})$ is the only graded prime ideal of $R$. Let $P$ be a graded semi-primary ideal of $R$. Then $\operatorname{Grad}(P)$ is a graded prime ideal of $R$ by Proposition 1.1, and then $\operatorname{Grad}(P)=\operatorname{Grad}(\{0\})$ which is a graded maximal ideal by Lemma 1.11 , and hence $P$ is a graded primary ideal of $R$ by ([11], Proposition 1.11).

Corollary 1.13. Let $R$ be a graded ring such that every homogeneous element is either unit or nilpotent. Then every graded semi-primary ideal of $R$ is graded 1-absorbing primary.
Theorem 1.14. Let $R$ be a graded local ring with a graded maximal ideal $X$. If $\operatorname{Grad}(\{0\})$ and $X$ are the only graded prime ideals of $R$, then every graded semi-primary ideal of $R$ is graded 1-absorbing primary.

Proof. Let $P$ be a graded semi-primary ideal of $R$. Then $\operatorname{Grad}(P)$ is a graded prime ideal of $R$ by Proposition 1.1. If $\operatorname{Grad}(P)=X$, then $P$ is a graded primary ideal of $R$ by ([11], Proposition 1.11), and hence $P$ is graded 1-absorbing primary. Suppose that $\operatorname{Grad}(P)=\operatorname{Grad}(\{0\})$. Let $x, y, z \in h(R)$ be nonunit elements such that $x y z \in P$ and $z \notin \operatorname{Grad}(\{0\})$. Then $x \in \operatorname{Grad}(\{0\})$ or $y \in \operatorname{Grad}(\{0\})$, and then $x y=0 \in P$ in both cases. Therefore, $P$ is a graded 1 -absorbing primary ideal of $R$.

Theorem 1.15. Let $R$ be a graded ring such that every homogeneous element is either unit or nilpotent. Then every proper graded ideal of $R$ is graded 1-absorbing primary.

Proof. Let $P$ be a proper graded ideal of $R$ and $x \in P$. Then $x_{g} \in P$ for all $g \in G$, and then $x_{g}$ is nilpotent for all $g \in$ $G$, which yields that $x_{g} \in \operatorname{Grad}(\{0\})$ for all $g \in G$, so $x \in$ $\operatorname{Grad}(\{0\})$. So, $P \subseteq \operatorname{Grad}(\{0\})$, and then $\operatorname{Grad}(\{0\}) \subseteq$ $\operatorname{Grad}(P) \subseteq \operatorname{Grad}(\{0\})$, that is $\operatorname{Grad}(P)=\operatorname{Grad}(\{0\})$ which is graded prime by Lemma 1.11 , and hence $P$ is a graded semi-primary ideal of $R$ by Proposition 1.1. Therefore, $P$ is a graded 1-absorbing primary ideal of $R$ by Corollary 1.13 .

The intersection of two graded prime ideals is not necessarily to be a graded prime ideal, see ([6], Example 5). However, if the intersection is also graded prime, then the two graded prime ideals should be comparable as one can see in the following lemma:

Lemma 1.16. Let $R$ be a graded ring and $P, K$ be two graded prime ideals of $R$. Then $P \cap K$ is a graded prime ideal of $R$ if and only if $P \subseteq K$ or $K \subseteq P$.

Proof. Suppose that $P \bigcap K$ is a graded prime ideal of $R$. Assume that $P \nsubseteq K$ and $K \nsubseteq P$. Then there exist $x \in P$ and $y \in K$ such that $x \notin K$ and $y \notin P$, and then $x_{g} \notin K$ and $y_{h} \notin P$ for some $g, h \in G$. Note that $x_{g} \in P$ and $y_{h} \in K$ as $P$ and $K$ are graded ideals. So, $x_{g} y_{h} \in P \bigcap K$, and then either $x_{g} \in P \bigcap K$ or $y_{h} \in P \cap K$, which is a contradiction. Therefore, $P \subseteq K$ or $K \subseteq P$. The converse is clear.

Theorem 1.17. Let $R$ be a graded ring. If every graded ideal of $R$ is graded 1-absorbing primary, then graded prime ideals of $R$ are comparable.

Proof. Let $P$ and $K$ be two graded prime ideals of $R$. Then $P \cap K$ is a graded ideal of $R$ by Proposition 0.1 , and then $P \cap K$ is a graded 1-absorbing primary ideal of $R$ by assumption, which implies that $\operatorname{Grad}(P \cap K)=P \bigcap K$ is a graded prime ideal of $R$ by Corollary 1.3. Hence, $P \subseteq K$ or $K \subseteq P$ by Lemma 1.16 .

Corollary 1.18. Let $R$ be a graded ring. If every graded ideal of $R$ is graded 1-absorbing primary, then $R$ is a graded local ring. 
Definition 1.19. [7] Let $R$ be a graded ring.

1. For $a, b \in h(R)$, we say that a divides $b$ (we write $a \mid b$ ) if $b=$ ax for some $x \in h(R)$.

2. $R$ is said to be a graded divided ring if for every graded prime ideal $P$ of $R$ and for every $a \in h(R)-P$, we have $a \mid p$ for every $p \in P$.

Proposition 1.20. [7] Let $R$ be a graded divided ring. Then every graded 1-absorbing primary ideal of $R$ is graded primary.

Proof. Suppose that $P$ is a graded 1-absorbing primary ideal of $R$. Let $a, b \in h(R)$ such that $a b \in P$ and $b \notin \operatorname{Grad}(P)$. We may assume that $a, b$ are nonunit elements of $R$. Since $\operatorname{Grad}(P)$ is a graded prime ideal of $R$ by Corollary 1.3 and $b \notin \operatorname{Grad}(P)$, we have that $a \in \operatorname{Grad}(P)$. Since $R$ is a graded divided ring, we have that $b \mid a$, which means that $a=b w$ for some $w \in h(R)$. Since $b \notin \operatorname{Grad}(P)$ and $a \in \operatorname{Grad}(P)$, we achieve that $w$ is a nonunit element of $R$. Since $a b=b w b \in P$ and $P$ is a graded 1-absorbing primary ideal of $R$ and $b \notin$ $\operatorname{Grad}(P)$, we have that $a=b w \in P$. Thus, $P$ is a graded primary ideal of $R$.

The next result gives another case in which every graded 1-absorbing primary ideal of $R$ is graded primary:

Proposition 1.21. Let $R$ be a graded local ring with a graded maximal ideal generated by a homogeneous element. Then every graded 1-absorbing primary ideal of $R$ is graded primary.

Proof. Let $M=R a$ be the graded maximal ideal of $R$, where $a \in h(R)$. Suppose that $P$ is a graded 1-absorbing primary ideal of $R$. Then $\operatorname{Grad}(P)$ is a graded prime ideal of $R$ by Corollary 1.3. Assume that $x, y \in h(R)$ such that $x y \in P$ and $y \notin \operatorname{Grad}(P)$. Then $x \in \operatorname{Grad}(P)$. If $y$ is unit, then $x \in P$ and we are done. If $x$ is unit, then $y \in P$, and then $y \in \operatorname{Grad}(P)$, which is a contradiction. So, we assume that $x$ and $y$ are nonunit elements. Then $x \in M$, and then $x=r a$ for some $r \in R$. Clearly, $r \in h(R)$. If $r$ is unit, then $M=R a \subseteq R x \subseteq \operatorname{Grad}(P)$, and hence $\operatorname{Grad}(P)=M$, which gives that $P$ is a graded primary ideal of $R$ by ([11], Proposition 1.11). Suppose that $r$ is a nonunit element. Then ary $=x y \in P$, and then $\operatorname{ar} \in P$ since $P$ is graded 1-absorbing primary and $y \notin \operatorname{Grad}(P)$, which gives that $x \in P$. Therefore, $P$ is a graded primary ideal of $R$.

Let $R$ be a $G$-graded ring and $P$ be a graded ideal of $R$. Assume that $g \in G$ such that $P_{g} \neq R_{g}$. In [7], $P$ is said to be a $g$ 1-absorbing primary ideal of $R$ if whenever nonunit elements $x, y, z \in R_{g}$ such that $x y z \in P$, then $x y \in P$ or $z \in \operatorname{Grad}(P)$. Also, $P$ is said to be a $g$-primary ideal of $R$ if whenever $x, y \in R_{g}$ such that $x y \in P$, then either $x \in P$ or $y \in \operatorname{Grad}(P)$.

Proposition 1.22. [7] Let $R$ be a $G$-graded ring and $g \in G$. If $R$ has a g-1-absorbing primary ideal that is not a g-primary ideal, then the sum of every nonunit element of $R_{g}$ and every unit element of $R_{g}$ is a unit element of $R_{g}$.
Proof. Suppose that $P$ is a $g$-1-absorbing primary ideal of $R$ that is not a $g$-primary ideal of $R$. Hence, there exist nonunit elements $a, b \in R_{g}$ such that neither $a \in P$ nor $a \in \operatorname{Grad}(P)$. Let $w$ be a nonunit element of $R_{g}$. Since $w a b \in P$ and $P$ is a $g$-1-absorbing primary ideal of $R$ and $b \notin \operatorname{Grad}(P)$, we conclude that $w a \in P$. Let $u$ be a unit element of $R_{g}$. Suppose that $w+u$ is a nonunit element of $R_{g}$. Since $(w+u) a b \in P$ and $P$ is a $g$-1-absorbing primary ideal of $R_{g}$ and $b \notin \operatorname{Grad}(P)$, we conclude that $(w+u) a=w a+u a \in P$. Since $w a \in P$, we conclude that $a \in P$, which is a contradiction. Thus, $w+u$ is a unit element of $R_{g}$.

In view of Proposition 1.22, we have the following conclusion:

Corollary 1.23. [7] Let $R$ be a $G$-graded ring and $g \in G$. If $R_{g}$ has a nonunit element and a unit element whose sum is nonunit element in $R_{g}$, then a graded ideal $P$ of $R$ is a $g-1$ absorbing primary ideal of $R$ if and only if $P$ is a g-primary ideal of $R$.

Corollary 1.24. [7] Let $R$ be a G-graded ring. If $R$ has an $e$-1-absorbing primary ideal that is not an e-primary ideal, then $R_{e}$ is a local ring.

Proof. By Proposition 1.22, the sum of every nonunit element of $R_{e}$ and every unit element of $R_{e}$ is a unit element of $R_{e}$, and then by ([5], Lemma 1), $R_{e}$ is a local ring.

In view of Corollary 1.24, we have the following conclusion:

Corollary 1.25. Let $R$ be a graded ring such that $R_{e}$ is nonlocal ring and $P$ be a graded ideal of $R$. Then $P$ is an $e$ primary ideal of $R$ if and only if $P$ is an e-1-absorbing primary ideal of $R$.

Let $R$ be a $G$-graded ring and $P$ be a graded ideal of $R$. Assume that $g \in G$ such that $P_{g} \neq R_{g}$. In [1], $P$ is said to be a $g$-prime ideal of $R$ if whenever $x, y \in R_{g}$ such that $x y \in P$, then $x \in P$ or $y \in P$. We state the following:

Definition 1.26. Let $R$ be a $G$-graded ring and $P$ be a graded ideal of $R$. Assume that $g \in G$ such that $P_{g} \neq R_{g}$. Then $P$ is said to be a g-semi-primary ideal of $R$ if whenever $x, y \in R_{g}$ such that $x y \in P$, then $x \in \operatorname{Grad}(P)$ or $y \in \operatorname{Grad}(P)$.

Using the same technique that is used in the proof of Proposition 1.1, one can prove the following result:

Proposition 1.27. Let $R$ be a graded ring and $P$ be a graded ideal of $R$. Then $P$ is a e-semi-primary ideal of $R$ if and only if $\operatorname{Grad}(P)$ is an e-prime ideal of $R$.

Proposition 1.28. Let $R$ be a graded ring over which every e-semi-primary ideal of $R$ is $e$-1-absorbing primary and $P$ be a graded ideal of $R$ such that $P$ is an e-prime ideal of $R$ which is not a graded maximal ideal of $R$. Suppose that $X$ is a graded maximal ideal of $R$ with $P \varsubsetneqq X_{e}$ and $J$ is a proper graded ideal of $R$ with $\operatorname{Grad}(J)=P$. Then $P_{e}^{2} \subseteq J$. Moreover, $P_{e}\left(X_{e}-P\right) \subseteq J$. Furthermore, if $P_{e}=P$, then $P_{e} X_{e} \subseteq J$. 
Proof. Let $m \in X_{e}-P$ and $p, q \in P_{e}$. Then $\operatorname{Grad}\left(J+R_{e} p q m\right)=$ $P$, and then $J+R_{e}$ pqm is an $e$-semi-primary ideal of $R$ by Proposition 1.27 , and so $J+R_{e}$ pqm is an $e$-1-absorbing primary ideal of $R$ by assumption. Since $p q m \in J+R_{e} p q m$ and $m \notin P=\operatorname{Grad}\left(J+R_{e} p q m\right), p q \in J+R_{e} p q m$, and then there exists $r \in R_{e}$ such that $p q(1-r m) \in J$. Since $J$ is an $e-1$ absorbing primary ideal of $R$, either $p q \in J$ or $1-r m \in J$. If $1-r m \in J \subseteq \operatorname{Grad}(J)=P \subset X_{e} \subseteq X$, then $1 \in X$ as $r m \in X$, which is a contradiction. So, $p q \in J$ and hence $P_{e}^{2} \subseteq J$. Similarly, $\operatorname{Grad}\left(J+R_{e} p m^{2}\right)=P$, which gives that $J+R_{e} p m^{2}$ is an $e$-1-absorbing primary ideal of $R$. So, we will have $p m \in J$. Therefore, $P_{e}\left(X_{e}-P\right) \subseteq J$. Furthermore, let $x \in X_{e}$. If $x \notin P$, then $P_{e} x \subseteq P_{e}\left(X_{e}-P\right) \subseteq J$. If $x \in P=P_{e}$, then $P_{e} x \subseteq P_{e}^{2} \subseteq J$. Consequently, $P_{e} X_{e} \subseteq J$.

\section{References}

[1] R. Abu-Dawwas, E. Yıldız, Ü. Tekir, and S. Koç, On graded 1-absorbing prime ideals, Sao Paulo Journal of Mathematical Sciences, (2021), https://doi.org/10.1007/s40863-021-00218-3.

[2] F. A. A. Almahdi, M. Tamekkante and A. Mamouni, Rings over which every semi-primary ideal is 1-absorbing primary, Communications in Algebra, 48 (9) (2020), 38383845.

[3] K. Al-Zoubi and M. Al-Azaizeh, On graded weakly 2absorbing primary submodules, Vietnam Journal of Mathematics, 47 (2019), 297-307.

[4] K. Al-Zoubi and N. Sharafat, On graded 2-absorbing primary and graded weakly 2 -absorbing primary ideals, Journal of Korean Mathematical Society, 54 (2) (2017), 675684.

[5] A. Badawi and E. Y. Celikel, On 1-absorbing primary ideals of commutative rings, Journal of Algebra and Its Applications, (2020), DOI: 10.1142/S021949882050111X.

[6] M. Bataineh and R. Abu-Dawwas, On graded 2-prime ideals, Mathematics, (2021), https://doi.org/10.3390/math9050493.

[7] M. Bataineh and R. Dawwas, Graded 1-absorbing primary ideals, submitted.

[8] F. Farzalipour and P. Ghiasvand, On the union of graded prime submodules, Thai Journal of Mathematics, 9 (1) (2011), 49-55.

[9] C. Nastasescu and F. V. Oystaeyen, Methods of graded rings, Lecture Notes in Mathematics, 1836, SpringerVerlag, Berlin, (2004).

${ }^{[10]}$ M. Refai and $R$. Abu-Dawwas, On generalizations of graded second submodules, Proyeccionese Journal of Mathematics, 39 (6) (2020), 1537-1554.

[11] M. Refai and K. Al-Zoubi, On graded primary ideals, Turkish Journal of Mathematics, 28 (3) (2004), 217-229.
[12] M. Refai, M. Hailat and S. Obiedat, Graded radicals and graded prime spectra, Far East Journal of Mathematical Sciences, (2000), 59-73.

[13] H. Saber, T. Alraqad and R. Abu-Dawwas, On graded $s$-prime submodules, Aims Mathematics, 6 (2020), 25102524.

[14] F. Soheilnia and A. Y. Darani, On graded 2-absorbing and graded weakly 2-absorbing primary ideals, Kyungpook Mathematical Journal, 57 (4) (2017), 559-580.

${ }^{[15]}$ R. N. Uregen, Ü. Tekir, K. P. Shum and S. Koç, On graded 2-absorbing quasi primary ideals, Southeast Asian Bulletin of Mathematics, 43 (2019), 601-613. 